\title{
Centro de Estudos e Pesquisa em Enfermagem (CEPEn): uma trajetória de 36 anos
}

\author{
Center of Studies and Research in Nursing (CEPEn): a trajectory of 36 years \\ Centro de Estudios y Investigación en Enfermería (CEPn): una trayectoria de 36 años
}

\begin{abstract}
Josete Luzia Leite
Diretora do Centro de Estudos e Pesquisa em Enfermagem, Associação Brasileira de Enfermagem, Brasília, DF.
\end{abstract}

Francisco Rosemiro Guimarães Ximenes Neto

Diretor de Assuntos Profissionais da Associação Brasileira de Enfermagem,

Brasília, DF.

rosemironeto@gmail.com

Isabel Cristina Kowal OIm Cunha

Diretora de Publicação e Comunicação Social da Associação Brasileira de Enfermagem, Brasília, DF.

Submissão: $10 / 10 / 2007$

Aprovação: 25/10/2007

\section{RESUMO}

O presente estudo trata dos destaques da trajetória do Centro de Estudos e Pesquisa em Enfermagem (CEPEn) da Associação Brasileira de Enfermagem no recorte temporal de 1971 a 2007. Estudo de cunho histórico social no qual utilizou-se o Catálogo Informações sobre Pesquisas e Pesquisadores em Enfermagem e os Relatórios dos Seminários Nacionais de Pesquisa em Enfermagem (SENPE). Os objetivos foram resgatar a trajetória do CEPEn e apresentar um dos eventos de maior importância para a Enfermagem Brasileira. A preocupação com o futuro e a necessidade de fortalecimento e enriquecimento, tanto dos docentes quanto dos profissionais do campo assistencial, é uma marca dos dias atuais e se coloca como desafio a ser enfrentado no presente.

Descritores: Pesquisa em enfermagem; História da enfermagem; Enfermagem.

\section{ABSTRACT}

The study presents some trajectory highlights of the Center of Studies and Research in Nursing of the Brazilian Nursing Association, considering the period from 1971 to 2007. This is a historical study based on the Information Catalog of Research and Researchers in Nursing and the reports of the National Seminars of Nursing Research. The objectives were to rescue the contribution of CEPEn and to present one of the most important events for Brazilian Nursing. The concern with the future and the need for empowerment and enrichment, of professors as well as for assistance professionals, is one of the marks in nowadays and it is a challenge to be faced in present time.

Descriptors: Nursing research; History of nursing; Nursing.

\section{RESUMEN}

El presente estudio trata de los realces de la trayectoria del Centro de Estudios y Investigación en Enfermería (CEPEn) de la Asociación Brasileña de Enfermería en el periodo de 1971 al 2007. Es un estudio histórico el lo cual se utilizó el Catalogo de Informaciones sobre Investigaciones y Investigadores y los reportes de los Seminarios Nacionales de Investigación en Enfermería. Los objetivos fueran rescatar la trayectoria del CEPEn y presentar uno de los eventos de más importancia para la Enfermería Brasileña. La preocupación con el futuro y la necesidad de fortalecimiento y enriquecimiento, tanto de los profesores como de los profesionales del campo asistencial es una marca de los días actuales y se pone como un desafío a ser enfrentado en la actualidad.

Descriptores: Investigación en enfermería; Historia de la enfermería, Enfermería.

Leite JL, Ximenes Neto FRG, Cunha ICKO. Centro de Estudos e Pesquisa em Enfermagem (CEPEn): uma trajetória de 36 anos. Rev Bras Enferm 2007 nov-dez; 60(6): 621-6.

\section{INTRODUÇÃO}

O CEPEn desde sua origem destina-se a incentivar o desenvolvimento e divulgação da pesquisa em Enfermagem, organizar e preservar documentos históricos da profissão e rege-se pelas disposições do Estatuto da Associação Brasileira de Enfermagem-ABEn Nacional e por Regimento próprio.

A constituição do CEPEn tem como pilar, a proposta elaborada por Dra. Haydée Guanais Dourado, da Comissão de Legislação da ABEn, aprovada na Assembléia de Delegados do XXIII Congresso Brasileiro de Enfermagem em Manaus, 1971 ${ }^{(1)}$. A Diretoria daABEn reunida em 1978, propôs a realização de um Seminário de Pesquisa em Enfermagem-SENPE que veio a tornar-se um dos eventos da maior importância e significado para o calendário científico da Enfermagem Brasileira. Com este propósito, em novembro de 1979 foi realizado o I SENPE, sediado pela Escola de Enfermagem de Ribeirão Preto-SP, cuja temática central foi O Estado Atual da Pesquisa em Enfermagem no Brasil. Foi um Seminário do qual participaram 40 enfermeiros/docentes, indicados pelas Escolas de Enfermagem de todo o país, conforme solicitado 
pela ABEn. A caracterização deste evento pioneiro esteve na participação intencional de pesquisadores de Saúde, vinculados a abordagens fortalecidas nas Ciências Humanas e Sociais. Algumas produções de conhecimento em dissertações de Mestrado, à época, foram apresentadas por seus autores e focalizadas como objeto de debate, no olhar ampliado das Ciências Sociais. Foi uma primeira sinalização coletiva da crítica ao modelo biologicista e a inquietação de seu dimensionamento social(2).

A partir deste, os demais SENPE, foram realizados regularmente a cada dois anos.

\section{METODOLOGIA}

Considerando o objetivo deste estudo, trata-se de uma pesquisa de cunho histórico social, realizada em 2007. Os critérios de inclusão foram: selecionaram-se os Anais do XXIII CBEN-Manaus, os catálogos "Informações sobre Pesquisas e Pesquisadores em Enfermagem" e, LivrosPrograma dos quatorze eventos do SENPE. Após esta seleção, foi feita leitura atenta, seguida de releitura de cada item, anotando-se os dados importantes para o estudo.

\section{RESULTADOS}

\section{Marcos Históricos dos SENPEs}

No primeiro marco - de 1979 a 1985 - ocorreram quatro SENPE: Ribeirão Preto (1979), Brasília (1982), Florianópolis (1984) e São Paulo (1985). Em Ribeirão Preto(2), objetivou-se conhecer e sintetizar a opinião dos participantes sobre áreas prioritárias e limitações da pesquisa em Enfermagem; o debate foi aberto com quarenta enfermeiros engajados em pesquisa e a tônica foi a metodologia e linhas gerais de pesquisa. O de Brasília( ${ }^{(3)}$, em 1982, reuniu 30 convidados, envolvidos com a pós-graduação e pesquisa de enfermagem, ainda dispersas em suas buscas, e muito incipiente nas relações com as políticas e prioridades de pesquisa, em andamento nos órgãos de apoio à investigação científica, no âmbito federal, representados pelo Conselho Nacional de Desenvolvimento Científico e Tecnológico do Ministério da Ciência e Tecnologia - CNPq/MCT e a Coordenadoria de Aperfeiçoamento de Pessoal do Ensino Superior do Ministério da Educação - CAPES/MEC. Ressalte-se que nesta década (1972) foi criado o primeiro Curso de Mestrado, na Escola de Enfermagem Anna Nery da Universidade Federal do Rio de Janeiro - EEAN-UFRJ(4). Estava iniciando a vigência do III Plano Básico de Desenvolvimento da Ciência e Tecnologia - PBDCT, e o CNPQ e a Capes, congregaram esforços junto à ABEn através do CEPEn, para implementação de um debate sobre Prioridades e Linhas de Pesquisa, buscando as possibilidades de suas imbricações e propostas de convergência em desdobramentos deste PBDCT, nos interesses da pesquisa de Enfermagem ${ }^{(5)}$. Assim, embora houvesse prenúncios de mudança pela presença de enfermeiras com pós-graduação, atuantes na ABEn e lotadas temporariamente nesses Órgãos, desenvolvendo projetos especiais de redemocratização da educação e da pesquisa de Saúde/Enfermagem, a transição ainda não era tão clara e gerava inquietação para qualquer tomada de decisão ainda que houvesse fortes debates nesse SENPE. Afinal, em Brasília, no Auditório da Sede da ABEN, pesquisadores de Enfermagem discutiram sobre suas Prioridades e Áreas de pesquisas emergentes além de analisar a produção científica de Enfermagem existente até então, face ao $\mathrm{PBDCT} / \mathrm{CNPQ}^{(5)}$. No Seminário realizado em Florianópolis ${ }^{(6)}, 181$ enfermeiros, indicando uma franca ampliação do interesse e da participação de enfermeiros no restrito círculo acadêmico de pesquisadores em Enfermagem, foram realizados reflexões sobre alternativas de interpretação metodológica na formulação do conhecimento. Deve-se atentar para a consonância entre o debate profissional e o desenvolver da pós-graduação e da pesquisa no país que, naquele momento, vivia as primeiras movimentações para encontrar a brecha para o fim do ciclo ditatorial do governo militar. Nesse período, um número crescente de enfermeiras já discutia questões teóricas concernentes à pesquisa em Enfermagem, bem como um maior número de enfermeiras já expressava a necessidade de uma base científica e suportes teórico-filósoficos para a realização das suas práticas, levantam as limitações e dificuldades da pesquisa ressaltando a crítica, mais evidente à falta de financiamento e as pressões das instituições para a produção do conhecimento em quantidade em detrimento da qualidade. O realizado em São Paulo ${ }^{(7)} \mathrm{em} \mathrm{1985,}$ aconteceram cinco simpósios e uma mesa redonda intitulada:ética e pesquisa.Os simpósios foram assim denominados:Tendências da pesquisa em Enfermagem materno-infantil,psiquiátrica,comunitária e médico-cirúrgica; utilização de alternativas metodológicas na pesquisa em Enfermagem; perfil do pesquisador enfermeiro: relato de experiência; ampliação do espaço para pesquisa em enfermagem e pesquisa em saúde: panorama da pesquisa em serviços de saúde na América Latina e incorporação dos resultados da pesquisa na prática de saúde . O segundo marco - 1988 a 1995 - destaca-se pela aproximação entre a pesquisa acadêmica e o cotidiano da prática profissional. Nesse período também foram realizados quatro SENPES: Belo Horizonte $^{(8)}(1988)$ cujo tema central foi "Pesquisa e Serviço de Enfermagem"; no Rio de Janeiro(9), em 1991, com o tema "trabalho e pesquisa", contou com a participação de 300 enfermeiros que discutiram sobre a pesquisa enquanto parte integrante do processo de trabalho do enfermeiro docente e de serviço, bem como os fatores a ela relacionados. Em Fortaleza ${ }^{(10)}(1994)$ foi explorado o tema "Pesquisa, ensino e assistência: o desafio profissional - e, novamente o SENPE é sediado em Ribeirão Preto(11) (1995) com o tema "A pesquisa no cotidiano da Enfermagem, a complexidade das relações". Aqui não escapa a vinculação medular com o novo momento democrático experimentado pelo Brasil, bem como a reverberação - notadamente na área da Saúde - da recém-ocorrida VIII Conferência Nacional de Saúde, que recoloca em debate, os princípios da Nova Reforma Sanitária e a Saúde como um direito universal, o que termina consagrado na Constituição Federal de 1988, denominada Constituição Cidadã ${ }^{(12)}$.

Houve um aumento quantitativo de pesquisadores na área de Enfermagem, em conseqüência direta da política de qualificação profissional e do novo papel assumido pela academia. Como conseqüência, as lideranças da Enfermagem levantaram questões e preocupações relativas aos tipos de pesquisa e a sua orientação filosófica.

O terceiro marco - iniciado em 1997 - delimita, mais claramente, os impasses, desafios e necessidades com os quais a Enfermagem - tanto em seu corpus profissional quanto acadêmico - se defronta a partir do enxugamento, por parte do Estado, das políticas de saúde e do redimensionamento do papel da universidade e da pesquisa, no quadro nacional. Busca-se, nesse momento, o fortalecimento e o enriquecimento, tanto dos que ensinam, quanto daqueles inseridos no campo profissional dos que atuam em Serviços de Saúde, ou mesmo em assessorias ou consultorias temporárias em Órgãos nas três esferas das políticas governamentais. Cinco SENPEs ocorreram nesse período: Vitória ${ }^{(13)}(1997), G^{2}$ ramado(14) $(1999)$, Belém $^{(15)}$ (2001), Porto Seguro(16) (2003), e São Luis ${ }^{(17)}$ (2005). E, novamente em Florianópolis ${ }^{(18)}(2007)$. As discussões nestes Seminários foram centradas nas necessidades da profissão, enfatizada sua articulação com a sociedade, com os temas: "Diretrizes para pesquisa em Enfermagem"; "A interdependência do cuidar e do pesquisar na Enfermagem"; "A pesquisa no espaço da Enfermagem: multiplicidade e complexidade"; "Interface da pesquisa em Enfermagem: aproximando o ensino e o cuidado com outros campos do conhecimento"; "A pesquisa em Enfermagem e sua expressão na atenção à saúde" e "Políticas de pesquisa em Enfermagem" segundo a ordem cronológica citada ${ }^{(5)}$. Este marco tem se caracterizado pelo desafio da democratização da pesquisa e com esta, o esforço do desenvolvimento de uma base científica de conhecimentos para a prática da profissão. Constatase que o número de pesquisadores de enfermagem vem aumentando, a organização de Grupos de Pesquisa, solidificando-se e possibilitando o maior intercâmbio entre grupos de pesquisa; por conseguinte, a disseminação do conhecimento,mobiliza-se em alguns importantes avanços ,a serem estudados, do ponto de vista, enfaticamente, qualitativo. 


\begin{tabular}{|c|c|c|}
\hline ORDEM & ANO & EIXOS TEMÁTICOS/MODALIDADE DISCUSSÃO \\
\hline & & Conferência: Estado atual da pesquisa no país \\
\hline \multirow[t]{4}{*}{$1^{0}$} & \multirow[t]{4}{*}{$\begin{array}{c}1979 \\
\text { Ribeirão Preto-SP(2) }\end{array}$} & $\begin{array}{l}\text { Plenária/pesquisa: } \\
\text { 1.Observação da administração de insulina em pacientes no domicílio } \\
\text { 2. A importância da imagem corporal na prática de enfermagem } \\
\text { 3. A enfermeira como coordenadora da assistência ao paciente }\end{array}$ \\
\hline & & Grupos de trabalho: Prioridades e limitações da pesquisa em enfermagem no país \\
\hline & & Painel: Prioridades e limitações da Pesquisa em Enfermagem no país \\
\hline & & NÃO HOUVE DIVULGAÇÃO EM ANAIS DE RESUMOS DE TRABALHOS APRESENTADOS ORAL/PÔSTER \\
\hline \multirow[t]{4}{*}{$2^{\circ}$} & \multirow{4}{*}{$\begin{array}{c}1982 \\
\text { Brasília-DF(3) }\end{array}$} & Conferência: Pesquisa em Enfermagem face ao Plano Básico de Desenvolvimento Científico e Tecnológico - PBDCT \\
\hline & & $\begin{array}{l}\text { Plenária/conferência: } \\
\text { 1.Situação atual dos Cursos de Pós-Graduação e a produção científica de Enfermagem e o impacto social de sua produção } \\
\text { 2. Pesquisa isolada e programas integrados de pesquisa } \\
\text { 3. Vazios do conhecimento e sugestões de temáticas relevantes na área da Enfermagem } \\
\text { 4. Perspectivas de pesquisa em Enfermagem } \\
\text { 5. Núcleos emergentes de pesquisa e Pós graduação em Enfermagem } \\
\text { 6. Divulgação do conhecimento produzido na área de Enfermagem }\end{array}$ \\
\hline & & $\begin{array}{l}\text { Trabalho de grupo: } \\
\text { 1.Situação da Pesquisa em Enfermagem face ao desenvolvimento social. Enfoque: linhas de pesquisa em enfermagem } \\
\text { 2.Proposta de perspectiva do ensino de Pós-graduação e a Pesquisa em Enfermagem no país para os próximos cinco anos }\end{array}$ \\
\hline & & Plenária: Estabelecimento de prioridades de pesquisa em enfermagem (Não houve divulgação de resumos de trabalhos oral/pôster) \\
\hline \multirow[t]{6}{*}{$3^{\circ}$} & \multirow{6}{*}{$\begin{array}{c}1984 \\
\text { Florianópolis-SC(6) }\end{array}$} & Conferência: a pesquisa e a prática de Enfermagem \\
\hline & & $\begin{array}{l}\text { Simpósio: o saber, a saúde e a pesquisa em Enfermagem } \\
\text { - Tema 1: a teoria do conhecimento } \\
\text { - Tema 2: alternativas metodológicas de pesquisa } \\
\text { - Tema 3: a construção do saber na Enfermagem: evolução histórica } \\
\text { - Tema 4: políticas de saúde }\end{array}$ \\
\hline & & $\begin{array}{l}\text { Painel: Abordagem metodológica } \\
\text { - Tema 1: o positivismo, o neopositivismo e o funcionalismo } \\
\text { - Tema 2: a antropologia e a fenomenologia } \\
\text { - Tema 3: o método dialético } \\
\end{array}$ \\
\hline & & $\begin{array}{l}\text { Simpósio: as teorias de enfermagem } \\
\text { - Tema 1: o marco teórico em trabalhos de pesquisa } \\
\text { - Tema 2: o surgimento e a evolução histórica das teorias de Enfermagem } \\
\text { - Tema 3: avaliação do estado das teorias de Enfermagem } \\
\end{array}$ \\
\hline & & $\begin{array}{l}\text { Mesa redonda: } \\
\text { 1.Situação da Enfermagem nos países representantes no Seminário } \\
\text { 2.Da representação da Enfermagem nos órgãos financiadores de pesquisa }\end{array}$ \\
\hline & & Painel: Pós-graduação para enfermeiros no Brasil (Não houve divulgação de resumos de trabalhos oral/pôster) \\
\hline \multirow[t]{4}{*}{$4^{\circ}$} & \multirow{4}{*}{$\begin{array}{c}1985 \\
\text { São Paulo-SP(7) }\end{array}$} & Simpósio: Tendências da pesquisa em Enfermagem: materno-infantil, psiquiátrica, comunitária e médico-cirúrgica \\
\hline & & $\begin{array}{l}\text { Simpósio: } \\
\text { 1.Utilização de alternativas metodológicas na pesquisa em Enfermagem } \\
\text { 2.Perfil do pesquisador enfermeiro: relato de experiência } \\
\text { 3.Ampliação do espaço para pesquisa em Enfermagem } \\
\text { 4.Pesquisa em saúde: panorama da pesquisa em serviços de saúde na América Latina e incorporação dos resultados da pesquisa } \\
\text { na prática de Enfermagem }\end{array}$ \\
\hline & & Mesa redonda: ética e pesquisa em Enfermagem \\
\hline & & NÃO HOUVE DIVULGAÇÃO DE TRABALHOS ORAL/PÔSTER \\
\hline \multirow[t]{4}{*}{$5^{\circ}$} & \multirow{4}{*}{$\begin{array}{c}1988 \\
\text { Belo Horizonte-MG(8) }\end{array}$} & Conferência: História da ciência \\
\hline & & Mesa redonda: saúde, prática e pesquisa \\
\hline & & Discussão de grupo: o enfermeiro, a pesquisa e a prática \\
\hline & & $\begin{array}{l}\text { Apresentação de estudo: As tendências em pesquisa sobre a prática em seis países da América Latina (Não houve divulgação de } \\
\text { resumos de trabalhos oral/pôster) }\end{array}$ \\
\hline \multirow[t]{3}{*}{$6^{\circ}$} & \multirow[t]{3}{*}{$\begin{array}{c}1991 \\
\text { Rio de Janeiro-RJ(9) }\end{array}$} & $\begin{array}{l}\text { Discursos de Abertura e Encerramento das } \\
\text { Presidente da ABEn-Nacional } \\
\text { Presidente da ABEn-RJ } \\
\text { Presidente do CEPEn } \\
\text { (Nos ANAIS do não constam os títulos dos discursos) }\end{array}$ \\
\hline & & $\begin{array}{l}\text { Mesas Redondas: } \\
\text { 1- Pesquisa em Enfermagem-Impacto e perspectivas. } \\
\text { 2- A pesquisa como parte do processo de trabalho do Enfermeiro } \\
\text { 3- As questões de gênero no âmbito da Enfermagem }\end{array}$ \\
\hline & & Sessões Simultâneas, Tribunas livres e Oficinas \\
\hline \multirow[t]{2}{*}{$7^{\circ}$} & \multirow[t]{2}{*}{$\begin{array}{c}1994 \\
\text { Fortaleza-CE }\end{array}$} & $\begin{array}{l}\text { Conferências: Pesquisa- Ensino- Assistência: O desafio profissional } \\
\text { Discurso de Abertura da Presidente da ABEn-Nacional } \\
\text { Discurso de Abertura e Encerramento das Presidente da ABEn-CE e Presidente do CEPEn } \\
\text { (Nos ANAIS não constam os títulos dos discursos) }\end{array}$ \\
\hline & & $\begin{array}{l}\text { Mesa redonda: Pesquisa em Enfermagem no Brasil } \\
\text { Oficinas e Tribunas livres }\end{array}$ \\
\hline \multirow[t]{2}{*}{$8^{\circ}$} & \multirow[t]{2}{*}{$\begin{array}{c}1995 \\
\text { Ribeirão Preto-SP(11) }\end{array}$} & $\begin{array}{l}\text { Discursos de Abertura e Encerramento das Presidente da ABEn-Nacional } \\
\text { Presidente da ABEn-Regional-RP } \\
\text { Presidente do CEPEn } \\
\text { Cursos, Oficinas, Painéis }\end{array}$ \\
\hline & & $\begin{array}{l}\text { Mesa redonda: } \\
\text { A Pesquisa no cotidiano da Enfermagem } \\
\text { A pesquisa no Ensino de Graduação } \\
\text { A pesquisa no Ensino de Pós Graduação } \\
\text { Pesquisa no Serviço } \\
\text { Pesquisa nos Núcleos, Grupos e Parcerias } \\
\text { Relatos de Experiências }\end{array}$ \\
\hline
\end{tabular}




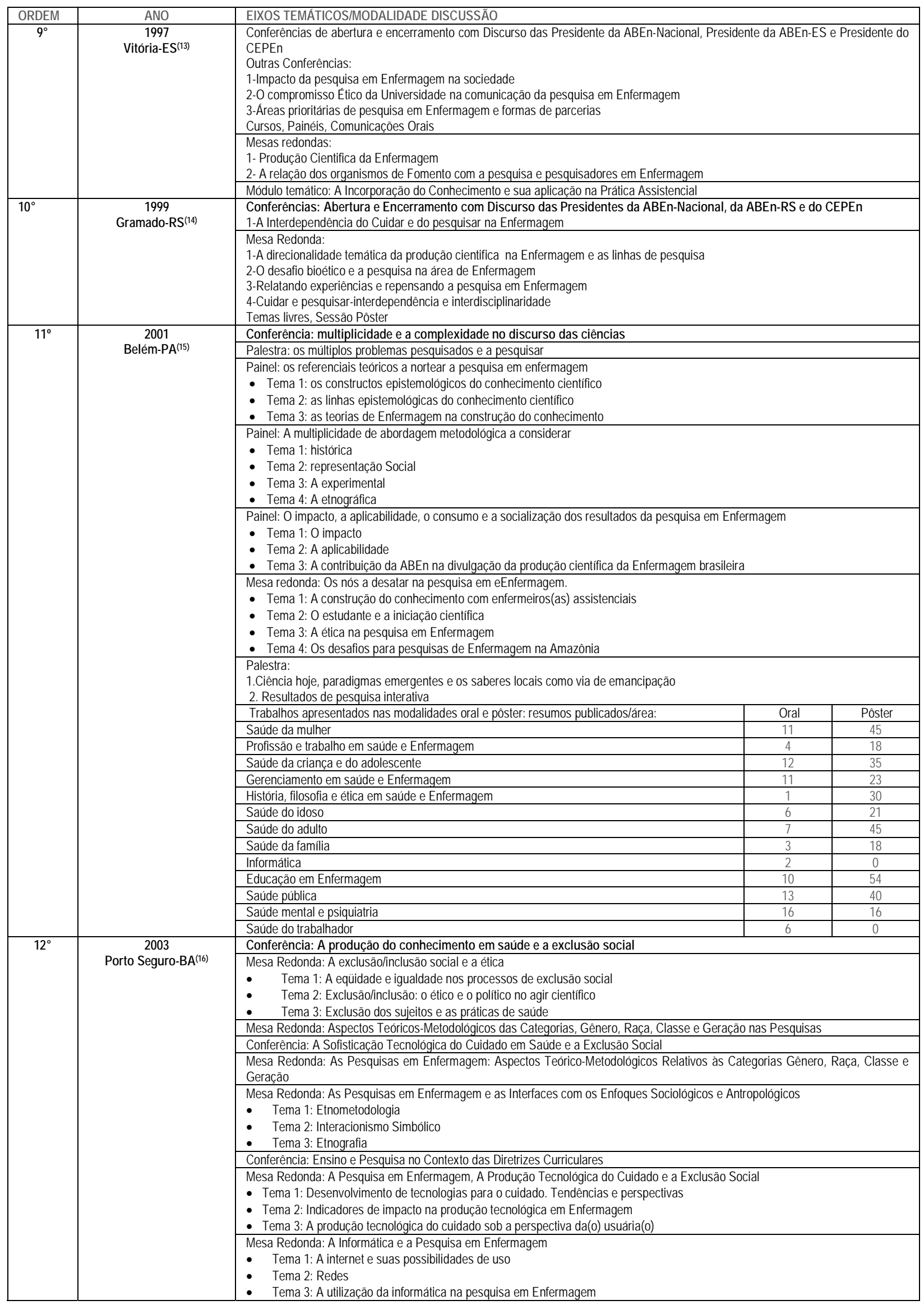




\begin{tabular}{|c|c|c|}
\hline ORDEM & ANO & EIXOS TEMÁTICOS/MODALIDADE DISCUSSÃO \\
\hline \multirow[t]{6}{*}{$12^{\circ}$} & \multirow{6}{*}{$\begin{array}{c}2003 \\
\text { Porto Seguro-BA(16) }\end{array}$} & Conferência: A Produção do Conhecimento em Saúde e a Exclusão Social \\
\hline & & $\begin{array}{l}\text { Mesa Redonda: A exclusão/inclusão social e a ética } \\
\text { - Tema 1: A eqüidade e igualdade nos processos de exclusão social: } \\
\text { - Tema 2: Exclusão/inclusão: o ético e o político no agir científico } \\
\text { - Tema 3: Exclusão dos sujeitos e as práticas de saúde }\end{array}$ \\
\hline & & Mesa Redonda: Aspectos Teóricos-Metodológicos das Categorias Gênero, Raça, Classe e Geração nas Pesquisas \\
\hline & & Conferência: A sofisticação tecnológica do cuidado em saúde e a exclusão social \\
\hline & & $\begin{array}{l}\text { Mesa Redonda: Produção e socialização do conhecimento em saúde } \\
\text { - Tema 1: Produção do conhecimento em Enfermagem } \\
\text { - Tema 2: Prioridades de pesquisas e o desenvolvimento científico e tecnológico em saúde } \\
\text { - Tema 3: A divulgação de informações da produção científica em Enfermagem }\end{array}$ \\
\hline & & $\begin{array}{l}\rightarrow \text { Lançamento de livros: } 5 \\
\rightarrow \text { Trabalhos apresentados modalidade pôster: } 289 \\
\rightarrow \text { Trabalhos apresentados modalidade oral/resumos publicados/área } \\
\text { - } \quad \text { Temas emergentes de investigação em enfermagem } \rightarrow 6 \\
\text { - } \quad \text { O cuidar de enfermagem como objeto de investigação } \rightarrow 14 \\
\text { - } \quad \text { A construção do conhecimento em enfermagem: reflexões teórico-filosóficas } \rightarrow 17 \\
\text { - } \quad \text { Conceitos e teorias na educação em enfermagem } \rightarrow 4 \\
\text { - } \quad \text { Técnicas de Observação e usos de imagens na pesquisa em enfermagem } \rightarrow 5 \\
\text { - } \quad \text { Representações sociais na pesquisa em enfermagem } \rightarrow 4 \\
\text { - } \quad \text { Abordagens fenomenológicas na pesquisa de enfermagem } \rightarrow 3 \\
\text { - } \quad \text { A informática na pesquisa, no ensino e na Assistência de enfermagem } \rightarrow 5 \\
\text { - } \quad \text { Análise qualitativa na pesquisa em enfermagem } \rightarrow 5 \\
\end{array}$ \\
\hline \multirow[t]{12}{*}{$13^{\circ}$} & \multirow{12}{*}{$\begin{array}{c}2005 \\
\text { São Luís-MA }\end{array}$} & Conferência: A produção e aplicação do conhecimento e a qualidade da atenção à saúde \\
\hline & & Painel: A saúde no Estado do Maranhão e São Luís: realidade, desafios e perspectivas \\
\hline & & $\begin{array}{l}\text { Painel: A pesquisa como ferramenta dinamizadora da prática profissional de Enfermagem } \\
\text { - Tema 1: Avanço da Pesquisa e da Prática Profissional } \\
\text { - Tema 2: Prioridade da Pesquisa na Saúde Coletiva } \\
\text { - Tema 3: Prioridade da Pesquisa Clínica }\end{array}$ \\
\hline & & Mesa Redonda: A pesquisa na formação de profissionais de saúde para o SUS/Enfermagem \\
\hline & & Conferência: Os modelos de atenção à saúde e os caminhos da pesquisa \\
\hline & & Conferência: Perfil de pesquisadores de Enfermagem no país \\
\hline & & $\begin{array}{l}\text { Painel: Diálogo entre os referenciais teóricos e as abordagens metodológicas } \\
\text { - Tema 1: Linhas epistemológicas do conhecimento } \\
\text { - Tema 2: Pluralidade da abordagem metodológica } \\
\text { - Tema 3: Instrumentos tecnológicos de apoio à Pesquisa em Saúde }\end{array}$ \\
\hline & & Mesa Redonda: As políticas de fomento à pesquisa em saúde e sua articulação com as políticas sociais \\
\hline & & Conferência: A produção do conhecimento em Enfermagem e sua expressão nos diversos campos de atenção à saúde \\
\hline & & $\begin{array}{l}\text { Mesa Redonda: A produção do conhecimento em Enfermagem e a educação popular em saúde hoje: pesquisa, extensão e ensino } \\
\text { na articulação com os movimentos populares }\end{array}$ \\
\hline & & $\begin{array}{l}\text { Painel: Compromisso da pesquisa em Enfermagem com a qualidade do processo de Assistência } \\
\text { - Tema 1: A agenda de prioridades da Pesquisa em Enfermagem } \\
\text { - Tema 2: A Pesquisa no território e ambiente da Saúde da Família } \\
\text { - Tema 3: A saúde de grupos populacionais vulneráveis } \\
\text { - Tema 4:Desenvolvimento de Tecnologias de Enfermagem para atenção à saúde }\end{array}$ \\
\hline & & $\begin{array}{l}\text { Trabalhos apresentados em Poster e Comunicação Oral/resumos publicados/área } \\
\text { - } \quad \text { Ensino } \rightarrow 30 \\
\text { - } \quad \text { Ética e Bioética } \rightarrow 25 \\
\text { - } \quad \text { Saúdo do Adolescente } \rightarrow 20 \\
\text { - } \quad \text { Saúde do Adulto } \rightarrow 92 \\
\text { - } \quad \text { Saúde Coletiva } \rightarrow 65 \\
\text { - } \quad \text { Saúde da Criança } \rightarrow 45 \\
\text { - } \quad \text { Saúde Mental da Mulher } \rightarrow 76 \\
\text { - } \quad \text { Saúde do Trabalhador } \rightarrow 35 \\
\text { - } \quad \text { Teses e Dissertações } \rightarrow 36 \\
\text { - } \quad \text { Multimídia } \rightarrow 9 \text { subáreas } \\
\end{array}$ \\
\hline \multirow[t]{3}{*}{$14^{\circ}$} & $\begin{array}{c}2007 \\
\text { Florianópolis-SC }{ }^{(18)}\end{array}$ & $\begin{array}{l}\text { Conferências: } \\
\text { 1- Abertura:Políticas de desenvolvimento científico-tecnológico e seu impacto nos espaços da Enfermagem nesse contexto } \\
\text { pesquisa em Enfermagem } \\
\text { 2- Encerramento: Agenda da ABEN na produção do conhecimento na Enfermagem }\end{array}$ \\
\hline & & $\begin{array}{l}\text { Mesas redondas: } \\
\text { Políticas de produção do Conhecimento em Enfermagem } \\
\text { Políticas de gerenciamento de pesquisa em Enfermagem } \\
\text { Políticas de formação de pesquisadores em Enfermagem }\end{array}$ \\
\hline & & $\begin{array}{l}\text { Sessões Coordenadas Simultâneas } \\
\text { Sessão de Pôster } \\
\text { Lançamento de livros }\end{array}$ \\
\hline
\end{tabular}

Quadro 1. SENPE - cronologia e temários. Brasília, 2007. 
Em junho de 2006 o Conselho Deliberativo do CNPq, que antes tinha apenas Representante de Área de Enfermagem, aprovou o ComitêAssessor de Enfermagem - CA-EF e, em outubro de 2006, a Enfermagem Brasileira registra a implantação deste Comitê considerado mais um avanço histórico para a Enfermagem, que vem se situando progressivamente, face as políticas públicas $^{(19)}$.

\section{CONSIDERAÇÕES FINAIS}

$\mathrm{Na}$ Enfermagem brasileira a pesquisa é uma atividade recente, no entanto há uma apreciável produção cientíica já consolidada. A preocupação crescente com o relativo distanciamento entre pesquisa e prática profissional é temática relevante, mas que exige conhecimento científico acadêmico e conhecimento científico aplicado, que não se esgota na pesquisa cientifica, mas a toma como parte de uma prática crescentemente qualificável. A pesquisa aplicada, recentemente acentuada como tal, em seu desenvolvimento na Enfermagem, nos denominados Mestrados Profissionais, reveste-se de importância para a prática profissional, mas exige, também, a superação das reservas, e porque não dizer do preconceito, ainda persistente, entre aqueles que insistem em posturas teoricistas e os que se apresentam em discursos praticistas. Ambos afastando-se da realidade da construção de uma prática crítico-criativa de mais suficiência em adequação qualitativa continuada ${ }^{(5)}$. 0 processo de retradução do conhecimento científico em validações desse conhecimento de feição tecnológica na prática cotidiana de enfermagem exige coletivo esforço de todos os profissionais para uma posição político-científica mais visível no processo de trabalho, tanto do pesquisador, quanto dos profissionais no campo da enfermagem. Do contrário,como se vê,há um vazio, falta uma ponte, no sentido de aproximar a teorização das situações concretas colocadas pelo campo de práticas profissionais; e, as respostas sem esta ponte, não só se torna lenta como possibilita seguir em sentido inverso, ao de se apropriar do conhecimento existente para enriquecer a prática profissional. Neste último aspecto, poderíamos perguntar: qual o valor atribuído, na prática profissional, à busca de estudos, a freqüência a biblioteca para a leitura de textos publicados nos periódicos brasileiros, a estudos de situações sistematizadas no próprio trabalho? Por outro lado, ainda poderíamos nos questionar sobre as pesquisas em desenvolvimento e a convergência dos estudos ao que se mostra como desafio nos campos de práticas profissionais? ${ }^{(17)}$

O CEPEn, até então, possui um acervo que demonstra, em parte, a construção científico-documental, produzida nas pesquisas ligadas a titulações acadêmicas. Dispõe atualmente, mais de 4.500 dissertações e teses, publicadas em catálogos até 2005 assim distribuídos: os volumes I a XVIII estão em CD-ROM e impressos, os demais, ou seja, de 2001 a 2005 também em CD-ROM e o de 2006, impresso para ser lançado no $59^{\circ}$ CBEn. Sabemos que uma profissão para exercer o seu papel precisa de uma entidade de classe que defenda todos os princípios da profissão com os olhos na história e na pujança de um presente e futuro arquitetado coletivamente. Desse modo, a ABEn vem se colocando como parceira dos usuários dos serviços profissionais da enfermagem no contexto da saúde e, na defesa de políticas sociais públicas transformadoras da nossa realidade.

É também por tudo isto que a ABEn, vive na incansável luta pela defesa dos interesses da categoria, nesses 81 anos, e o CEPEN nos seus 36 ao buscar as transformações, transformou-se. Essa transformação, de caráter inconclusivo, é acorde com uma sociedade, que necessariamente, está em constante mudança, em ritmo acelerado, exigindo agilidade de comunicação e proposições que atualizem as estratégias de luta, sem perder de vista os sonhos da profissão, suas tradições e seus preceitos éticos e legais.

A preocupação com o futuro e a necessidade de fortalecimento e enriquecimento, tanto dos docentes quanto dos profissionais do campo assistencial, é uma marca dos dias

\section{REFERÊNCIAS}

1. Associação Brasileira de Enfermagem. Relatório do $23^{\circ}$ Congresso Brasileiro de Enfermagem; 1971 jul 11-17; Manaus (AM), Brasil. Rev Bras Enferm 1971;24(5/6): 1-263.

2. Associação Brasileira de Enfermagem. Relatório do $1^{0}$ Seminário Nacional de Pesquisa em Enfermagem; 1979 nov 20-22; Ribeirão Preto (SP), Brasil. Ribeirão Preto (SP): ABEn/CNPq; 1979.

3. Associação Brasileira de Enfermagem. Relatório do $2^{\circ}$ Seminário Nacional sobre Ensino de Pós-Graduação e Pesquisa em Enfermagem; 1982 mar 24-26; Brasília (DF), Brasil. Brasília (DF) : ABEn/CNPq; 1982.

4. Leite JL, Oliveira DC. Centro de Estudos e Pesquisas em Enfermagem e a ABEn: 80 anos de desafios [editorial]. Rev Enferm UERJ 2006; 14(2): 161-2.

5. Leite JL, Paim L. A trajetória do Centro de Estudos e Pesquisas em Enfermagem. J ABEn 2006;48(4):18-20.

6. Associação Brasileira de Enfermagem. Anais do $3^{\circ}$ Seminário Nacional de Pesquisa em Enfermagem; 1984 abr 3-6; Florianópolis (SC), Brasil. Florianópolis (SC): ABEn-SC; 1984.

7. Associação Brasileira de Enfermagem. Anais do $4^{\circ}$ Seminário Nacional de Pesquisa em Enfermagem; 1985 set 2-5; São Paulo (SP), Brasil. São Paulo (SP): ABEn-SP; 1985.

8. Associação Brasileira de Enfermagem. Anais do $5^{\circ}$ Seminário Nacional de Pesquisa em Enfermagem; 1988 jul 25-29; Belo Horizonte (MG), Brasil. Belo Horizonte (MG): ABEn-MG; 1988.

9. Associação Brasileira de Enfermagem. Anais do $6^{\circ}$ Seminário Nacional de Pesquisa em Enfermagem; 1991 jul/ago 29-1; Rio de Janeiro (RJ), Brasil. Rio de Janeiro (RJ): ABEn-RJ; 1991.

10. Associação Brasileira de Enfermagem. Anais do $7^{\circ}$ Seminário Nacional de Pesquisa em Enfermagem; 1994 jun 5-9; Fortaleza (CE), Brasil. Fortaleza (CE): ABEn-CE; 1994.
11. Associação Brasileira de Enfermagem. Anais do $8^{\circ}$ Seminário Nacional de Pesquisa em Enfermagem; 1995 jul 11-13; Ribeirão Preto (SP), Brasil. Ribeirão Preto (SP): ABEn-SP; 1995.

12. Senado Federal (BR). Constituição: República Federativa do Brasil: 1988. São Paulo (SP): Atlas; 1988.

13. Associação Brasileira de Enfermagem. Anais do $9^{\circ}$ Seminário Nacional de Pesquisa em Enfermagem; 1997 jul 22-25; Vitória (ES), Brasil. Vitória (ES): ABEn-ES; 1997.

14. Associação Brasileira de Enfermagem. Anais do $10^{\circ}$ Seminário Nacional de Pesquisa em Enfermagem; 1999 maio 24-27; Gramado (RS), Brasil. Gramado (RS): ABEn-RS; 1999.

15. Associação Brasileira de Enfermagem. Anais do $11^{\circ}$ Seminário Nacional de Pesquisa em Enfermagem; 2001 maio 27-30; Belém (PA), Brasil [CD-ROM]. Belém(PA) : ABEn-PA; 2001.

16. Associação Brasileira de Enfermagem. Anais do $12^{\circ}$ Seminário Nacional de Pesquisa em Enfermagem; 2003 abr 27-30; Porto Seguro (BA), Brasil. Porto Seguro (BA): ABEn-BA; 2003.

17. Associação Brasileira de Enfermagem. Anais do $13^{\circ}$ Seminário Nacional de Pesquisa em Enfermagem; 2005 jun 14-17; São Luís (MA), Brasil [CD-ROM]. São Luís (MA): ABEn-MA; 2005.

18. Associação Brasileira de Enfermagem. Anais do $14^{\circ}$ Seminário Nacional de Pesquisa em Enfermagem; 2007 mai/jun 29-1; Florianópolis (SC), Brasil [CD-ROM]. Florianópolis (SC) : ABEnSC; 2007.

19. Ministério da Ciência e Tecnologia (BR). Conselho Nacional de Desenvolvimento Científico e Tecnológico. Comitê de Assessoramento de Enfermagem - CA-EF: composição-2006. [citado em 2007 mar 25]. Disponível em: URL: http:// www.cnpq.br/cas/ca-ef.htm 